\title{
The Influence of Load Eccentricity on the Stability of a Two-Member Column with PZT Rod
}

\author{
J. PRZYBYLSKI* AND K. KulińSKI \\ Częstochowa University of Technology, J.H. Dabrowskiego 73, 42-201 Częstochowa, Poland \\ Doi: $10.12693 /$ APhysPolA.138.203 \\ *e-mail: jacekpr@imipkm.pcz.pl
}

\begin{abstract}
In this paper the stability of a cantilever two-member column made of an aluminum host rod and a discretely mounted piezoceramic rod is discussed. Both rods are connected to each other at the column's free end by an ideally rigid mounting head. Knowing that in practical engineering it is impossible to achieve an ideal axiality of the load application and that the assembling of structure components may lead to an unintended mounting inaccuracy, the presence of the unintentional eccentricity of the axial load is taken into considerations. To prevent prebuckling of the column, the piezo rod is proposed to be mounted discretely with an offset distance in regard of the host column. Next, the static deflection and the internal axial force distribution are determined to estimate how the electric field application modifies the static behavior of the system resulting originally from the external force loading. During numerical calculation, the offset distance and the eccentricity of the external load are taken as control parameters to prove that piezo actuation is efficient for suppressing column's deformation when the external eccentric load is relatively small. It is also shown that column deflection may be reduced, reverted and even its rectilinear shape may be recovered.
\end{abstract}

topics: stability, load eccentricity, offset distance, buckling envelopes, piezoelectric actuation

\section{Introduction}

Piezoceramic materials are widely used in engineering to actively or passively enhance systems' stability and/or alter their natural vibration frequency via the piezoelectric actuation. In those applications the reverse piezoelectric effect is used, according to which the electric field acting on piezoceramic material deforms its shape. In this way, dependently on the electric field vector direction, a compressive or tensile force may be induced in the system.

Stiffening beam and column structures by elements made of piezoelectric materials and/or shape memory alloys (SMA) is studied and discussed by many researchers. Thompson and Loughlan [1] investigated the stability control and shape enhancement of carbon-epoxy columns, by embedding actuators to two adjacent and parallel external surfaces. Through the induction of a controlled value of voltage to the actuators, it was shown that the generated residual force could counteract the bending moment resulting from the external load applied to structure. It was stated that the application of a controlled voltage to the actuators may cause partial or even complete lateral deflection reduction of the structure. De Faria [2] as well as Zehetner and Irschik [3] studied the enhancement of buckling loads via the piezoelectric actuation in beams with supports preventing their longitudinal displacement. Colocally mounted piezoceramic patches, when supplied by the electric field, generated an axial force along the beam which counteracted the external axial compressive force. Przybylski and Sokó [4] investigated the reduction of lateral deflection in a simply supported beam with discretely attached piezoceramic rod subjected to eccentric load. The problem was formulated on the basis of stationary total potential energy principle with the use of von Karman theory concerning nonlinear strain-displacement relations. Performed numerical calculations proved that piezoelectric actuation may be successfully applied to eliminate the beam transversal displacement arising due to eccentric external load. Buckling and flutter instability of a non-conservative system in the form of a column with one end fixed and the second one restrained with a spring was discussed in [5]. It was shown in that paper that if some of the design parameters i.e. spring stiffness, mass ratio, follower force parameter exceeds a certain critical value, the column may undergo buckling and/or flutter instability.

It is worth noticing that the stability enhancement of slender structures via piezoelectric actuation is influenced not only by the introducted voltage and the length of piezoelements, but also by their location on the structure and the way in which those elements are attached. There are many studies, e.g. [6-8], concerning the optimization of various type of physical, geometrical and constructional parameters on shape and stability enhancement of different intelligent structures. 


\section{Formulation and solution of the problem}

In this paper, the problem concerns a slender cantilever two-member column with discretely attached piezoceramic (PZT) rod, shown in Fig. 1a. The first column member (host) is made of aluminium and is composed of two equidistantly located, aluminium rods with rectangular cross-section (see Fig. 1b). The second member, in the form of PZT rod is made of P-41 material (material properties taken according to the producer given information [9]) and is symmetrically located between two host column rods. Both column members are fixed to the ground with one end, whereas their other ends are connected by ideally rigid mounting head. Knowing that in practical engineering it is impossible to achieve an ideal axiality of loading, an eccentricity of the external force $P$ acting in a constant direction is taken into account. It is assumed that generation of the compressive or tensile piezoelectric force in the PZT rod, mounted with offset $d$ measured along $z$-axis in regard of the host member centroidal axis, enables one to significantly counteract the system's bending moment resulting from the eccentricity $\bar{e}$ of external load $P$.

The problem has been formulated on the basis of the variational principle of stationary total potential energy. In the formulation the linear constitutive equations for piezoelectric material and von Karman theory of small strains and moderate rotations with the nonlinear effect have been included. On the basis of von Karman theory, the straindisplacement relation in the $x$ direction and the member curvature can be expressed as

$$
\begin{aligned}
& \varepsilon(x)=\frac{\mathrm{d} U(x)}{\mathrm{d} x}+\frac{1}{2}\left[\frac{\mathrm{d} W(x)}{\mathrm{d} x}\right]^{2}, \\
& \kappa(x)=-\frac{\mathrm{d}^{2} W(x)}{\mathrm{d} x^{2}},
\end{aligned}
$$

where $\varepsilon(x)$ denotes the mid-plane strain, $\kappa(x)$ is the curvature and $U(x), W(x)$ denote the axial and transverse displacements, respectively.

The total energy potential stored in the system may be expressed as

$$
\begin{aligned}
V & =\frac{1}{2} \sum_{i=1}^{2} \int_{0}^{l} E_{i}\left[A_{i} \varepsilon_{i}^{2}+J_{i} \kappa_{i}^{2}\right] \mathrm{d} x \\
& -E_{z} A_{2}\left[e_{31} \int_{0}^{l} \varepsilon_{2} \mathrm{~d} x+\frac{1}{2} \xi_{33} E_{z} \int_{0}^{l} \mathrm{~d} x\right] \\
& +P U_{1}(l)+\left.P \bar{e} \frac{\mathrm{d} W_{1}(x)}{\mathrm{d} x}\right|_{x=l},
\end{aligned}
$$

where subscript $i=1,2$ denotes column's host and piezo member, respectively, $E_{i}$ - the Young modulus, $A_{i}$ - cross-section area of $i$-th member and $J_{i}-i$-th member substantial area moment of inertia with respect to the system's neutral axis, $E_{z}$ is the magnitude of the electric field vector applied across the thickness of the piezo rod (a)
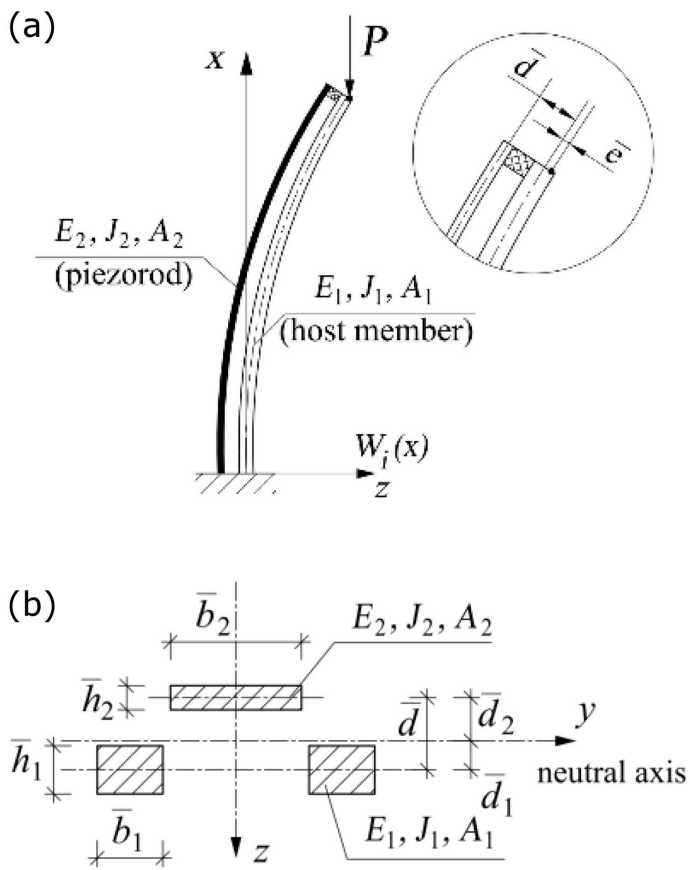

Fig. 1. Two-member column subjected to load $P$ with eccentricity $\bar{e}(\mathrm{a})$, column's cross-section (b).

(parallel to the 3 -axis), $e_{31}=d_{31} E_{2}, \xi_{33}$ stands for the dielectric constant of the piezoelectric material, $\bar{e}$ - external load eccentricity with respect to the host member centroidal axis.

The process of solution of Eq. (2) in regard of the non-conservative system with identical boundary conditions has been presented in detail in [10]. In order to increase the applicability of obtained results, based on methods presented in [10], the problem has been solved on the basis of non-dimensional parameters.

\section{Numerical results and discussion}

In this section the numerical results concerning the shape enhancement of the column subjected to eccentric axial load are presented. It should be noted that all the column's dimensions have been related to the non-dimensional host column member thickness $h_{1}$. The width of host member is $b_{1}=1.5 h_{1}$, PZT rod thickness $h_{2}=0.5 h_{1}$, its width $b_{2}=3 h_{1}$ and total column length $30 h_{1}$, respectively. Non-dimensional external load eccentricity parameter $e$ takes three values: $-0.01,0$ and 0.01. Specific location of the PZT member has been assumed, where offset $d=1 / 60$, which corresponds to the case where the PZT neutral axis coincides with the outer contour of the host rods. The host member is made of aluminium, for which the Young modulus $E_{1}=70.0 \mathrm{GPa}$, whereas PZT member is made of P-41 material for which $E_{2}=83.33 \mathrm{GPa}$ and the piezoelectric constant $e_{31}=8.333$. It has been assumed, in order to prevent depolarization of the PZT material, that the introduced electric field cannot exceed $2000 \mathrm{~V} / \mathrm{mm}$, which gives 


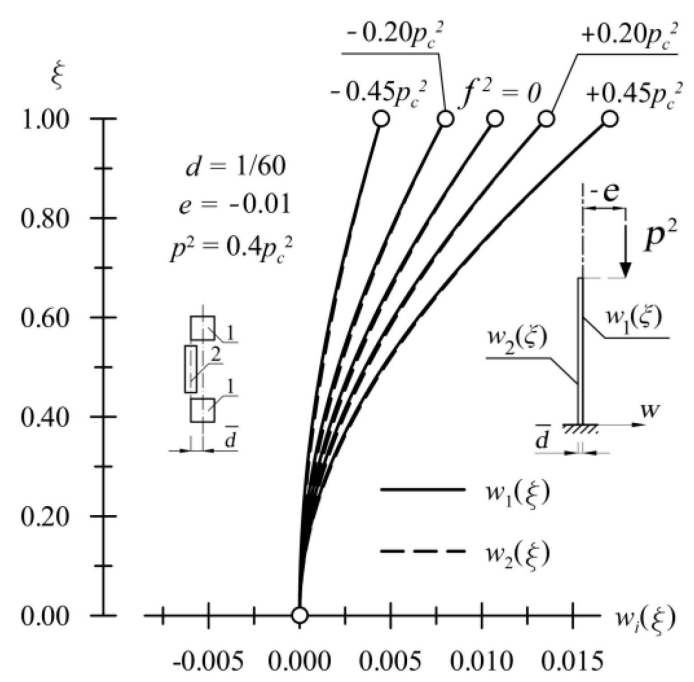

Fig. 2. Shape enhancement via piezoelectric actuation of a column subjected to axial load $p^{2}=0.4 p_{c}^{2}$ with eccentricity $e=-0.01$; other parameters: $d=1 / 60$.

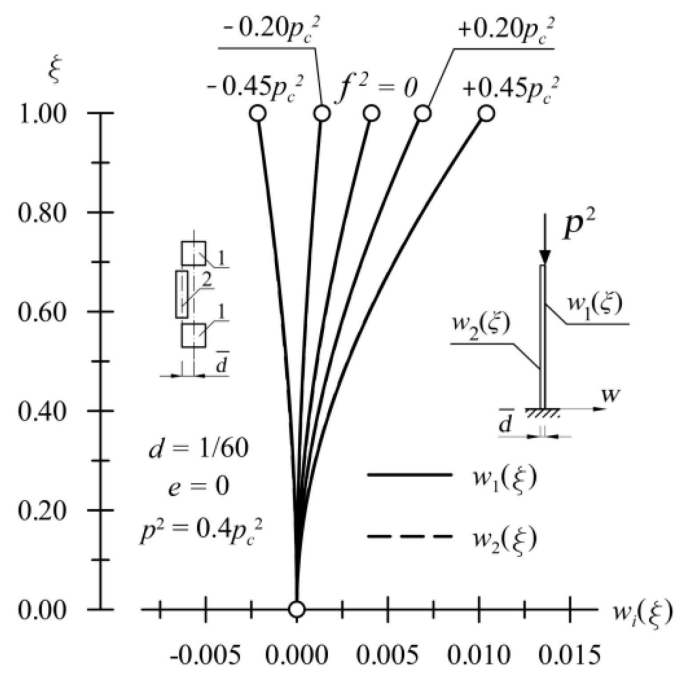

Fig. 3. Shape enhancement via piezoelectric actuation of a column subjected to pure axial load $p^{2}=0.4 p_{c}^{2}, e=0$; other parameters: $d=1 / 60$.

the maximum operational range of non-dimensional piezoelectric force $f^{2} \in\left\langle-0.45 p_{c}^{2}, 0.45 p_{c}^{2}\right\rangle$, where $p_{c}^{2}$ is the nondimensional critical buckling load for the analyzed system $\left(p_{c}^{2}=\pi^{2} / 4\right)$.

The columns deformation for the actuated and non-actuated systems subjected to load $p^{2}=0.4 p_{c}^{2}$ and different values of load eccentricity $e$ are presented in Figs. 2-4.

As shown in the figures, an application of the electric field to piezoceramic rod modifies the column deflection. In case of a column subjected to the axial load with $e=-0.01-$ the eccentricity measured in the opposite direction in regard to the PZT rod shift (see Fig. 2) - the column deflection may be significantly reduced, however, the

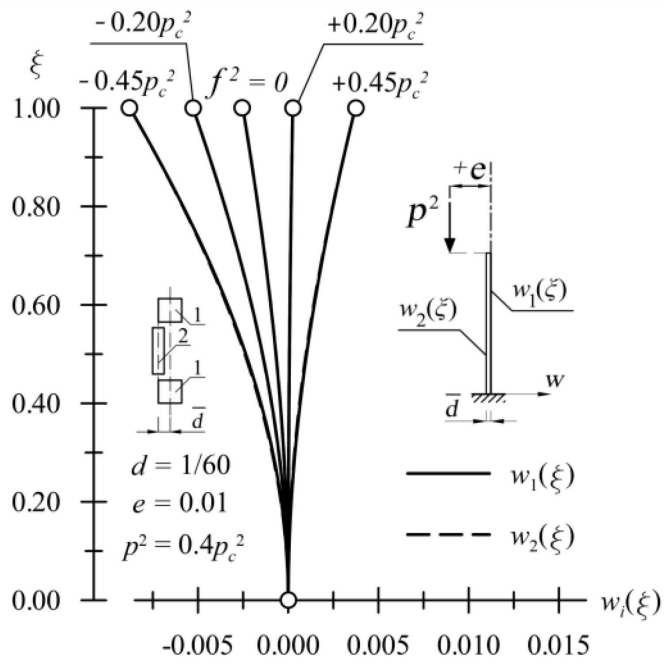

Fig. 4. Shape enhancement via piezoelectric actuation of a column subjected to axial load $p^{2}=0.4 p_{c}^{2}$ with eccentricity $e=0.01$; other parameters: $d=$ $1 / 60$.

rectilinear shape of the column cannot be achieved. In such case, harder PZT material should be used which may operate under larger electric field intensity or stack PZT elements should replace the piezoceramic rod. The stack transducer operates in $d_{33}$ working mode which characterizes greater performance. In the case of $e=0$ (Fig. 3), an induction of the compressive piezoelectric force allows one to obtain rectilinear column shape or even its deflection may be reverted. The closer the load is applied to the column's center of mass, the lower value of piezoelectric force is required to achieve the rectilinear column's shape.

In case of eccentricity $e=0.01$ (Fig. 4), the tensile piezoelectric force has to be induced to completely remove the column's deflection. It should be noted that with growing values of the external load, the range of the column's shape enhancement reduces.

\section{Conclusions}

The influence of piezoelectric force on the prebuckling behaviour of an eccentrically loaded column compound of a host aluminium rod and a PZT rod attached with an offset in regard of the first one is analytically modeled and thoroughly discussed on the basis of numerical analysis. It has been demonstrated that piezoelectric actuation is an effective tool for reducing the column's deformation resulting from the external load eccentricity. Dependently on the load eccentricity, properties of the PZT material and the offset value, the deflection may be significantly reduced, a rectilinear shape may be achieved or even the column's deflection may be reverted. Performed analysis proved that the reverse piezoelectric effect may be efficiently used for the shape control of slender systems. 


\section{Acknowledgments}

The authors gratefully acknowledge the financial support of Polish Ministry of Science and Higher Education (Grant No. BS/PB-1-101-3020/17/P).

\section{References}

[1] S. Thompson, J. Loughlan, Compos. Struct. 32, 59 (1995).

[2] A.R. de Faria, S.F. de Almeida, Compos. Struct. 65, 2 (2004).

[3] C. Zehetner, H. Irschik, Smart Struct. Syst. 4, 67 (2008).

[4] J. Przybylski, K. Sokół, Thin-Walled Struct. 49, 652 (2011).
[5] S.A. Fazelzadeh, M. Eghtesad, M. Azadi, Int. J. Struct. Stabil. Dynam. 10, 1083 (2010).

[6] S. Adali, I.S. Sadek, J.C. Bruch Jr., J.M. Sloss, Compos. Stuct. 71, 293 (2005).

[7] S. Choi, J.J. Lee, D.C. Seo, J. Compos. Mater. 34, 1494 (2000).

[8] E.P. Hadjigeorgiou, G.E. Stavroulakis, C.V. Massalas, Int. J. Eng. Sci. 44, 409 (2006).

[9] Annon, PZT Materials.

[10] J. Przybylski, K. Kuliński, Eng. Struct. 189, 644 (2019). 